\title{
SIEVING THE POSITIVE INTEGERS BY SMALL PRIMES
}

\section{A. GOLDSTON AND KEVIN S. MCCURLEY}

ABSTRACT. Let $Q$ be a set of primes that has relative density $\delta$ among the primes, and let $\phi(x, y, Q)$ be the number of positive integers $\leq x$ that have no prime factor $\leq y$ from the set $Q$. Standard sieve methods do not seem to give an asymptotic formula for $\phi(x, y, Q)$ in the case that $\frac{1}{2} \leq \delta<1$. We use a method of Hildebrand to prove that

$$
\phi(x, y, Q) \sim x f(u) \prod_{\substack{p \leq y \\ p \in Q}}\left(1-\frac{1}{p}\right)
$$

as $x \rightarrow \infty$, where $u=\frac{\log x}{\log y}$ and $f(u)$ is defined by

$$
u^{\delta} f(u)= \begin{cases}\frac{e^{\gamma \delta}}{\Gamma(1-\delta)}, & 0<u \leq 1, \\ \frac{e^{\gamma \delta}}{\Gamma(1-\delta)}+\delta \int_{0}^{u-1} f(t)(1+t)^{\delta-1} d t, & u>1 .\end{cases}
$$

This may also be viewed as a generalization of work by Buchstab and de Bruijn, who considered the case where $Q$ consisted of all primes.

1. Introduction. Let $Q$ be a set of primes, and let $\phi(x, y, Q)$ be the number of positive integers $\leq x$ that have no prime factor $\leq y$ from the set $Q$. In the case that $Q$ consists of all primes, it was proved by Buchstab [2] that

$$
\phi(x, y, Q) \sim x e^{\gamma} \omega(u) \prod_{p \leq y}\left(1-\frac{1}{p}\right) .
$$

where $u=\frac{\log x}{\log y}$ is fixed and $\omega(\cdot)$ is defined by

$$
u \omega(u)= \begin{cases}0, & 0<u \leq 1 \\ 1+\int_{0}^{u-1} \omega(t) d t, & u>1\end{cases}
$$

Uniform estimates for this case were later given by de Bruijn [1].

Our aim in the present paper is to give an asymptotic estimate for $\phi(x, y, Q)$ in the case that the set $Q$ has relative density $\delta$ among the primes, where $0<\delta<1$. If $Q$ is a set of primes, we define

$$
\psi(x, Q)=\sum_{\substack{p^{m} \leq x \\ p \in Q}} \log p,
$$

Received by the editors August 13, 1987 and, in revised form, September 25, 1987.

1980 Mathematics Subject (lassification (1985 Revision). Primary 11N25, 11 N35.

The research of the first author was supported by a grant from the National Science Foundation. The research of the second author was supported by a grant from the National Security Agency. 
For a set $Q$ of primes, we use $Q^{c}$ to denote the set of primes that are not in $Q$. Throughout the rest of this paper we shall assume that $Q$ has relative density $\delta$ among the primes, or more precisely that if $R(x)$ is defined by $\psi\left(x, Q^{c}\right)=$ $(1-\delta) x+x R(x)$, then there exists a constant $A>1$ such that

$$
R(x)=O\left(\frac{1}{\log ^{A} x}\right)
$$

For example, it follows from the prime number theorem for arithmetic progressions that if $Q$ consists of the primes belonging to a finite union of arithmetic progressions, then (1) is satisfied for every $A>0$.

In the case that $(1)$ is satisfied with $0<\delta<1$, estimates for $\phi(x, y, Q)$ can be obtained by a variety of sieve methods, and in particular it follows from a "fundamental lemma" (see [4, Theorem 2.5]) that

$$
\begin{aligned}
\phi(x, y, Q)=x W(y)(1 & +O(\exp (-\sqrt{\log x})) \\
& +O(\exp (-u(\log u-\log \log 3 u-\log \delta-2)))),
\end{aligned}
$$

where throughout this paper we shall write $u=\frac{\log x}{\log y}$ and

$$
W(y)=\prod_{\substack{p \leq y \\ p \in Q}}\left(1-\frac{1}{p}\right)
$$

If $u \rightarrow \infty$, then this gives an asymtotic estimate for $\phi(x, y, Q)$. When $u$ is constant (2) no longer gives an asymptotic for $\phi(x, y, Q)$, but in the case that $Q$ satisfies (1) with $0 \leq \delta<\frac{1}{2}$, Iwaniec [7] used the sieve of Eratosthenes-Legendre to prove a general sieve result that gives

$$
\phi(x, y, Q)=x W(y)\left(f(u)+O\left(\frac{u+1}{(1-2 \delta)(\log y)^{1-2 \delta}}\right)\right)
$$

where $f(\cdot)$ is defined by

$$
u^{\delta} f(u)= \begin{cases}\frac{e^{\gamma \delta}}{\Gamma(1-\delta)}, & 0<u \leq 1 \\ \frac{e^{\gamma \delta}}{\Gamma(1-\delta)}+\delta \int_{0}^{u-1} f(t)(1+t)^{\delta-1} d t, & u>1\end{cases}
$$

(Note the similarity to the Buchstab function when $\delta=1$.)

For the case when $\frac{1}{2} \leq \delta<1$, the Rosser Sieve [8] gives the best known result, namely that

$$
g_{1}(u) x W(y)(1+o(1)) \leq \phi(x, y, Q) \leq g_{2}(u) x W(y)(1+o(1)),
$$

where $g_{1}$ and $g_{2}$ are continuous solutions of

$$
\begin{array}{rlrl}
u^{\delta} g_{2}(u) & =A, \quad u \leq \beta+1, & \\
u^{\delta} g_{1}(u) & =B, \quad u \leq \beta, \\
\left(u^{\delta} g_{2}(u)\right)^{\prime} & =\delta u^{\delta-1} g_{1}(u-1), \quad u>\beta+1, \\
\left(u^{\delta} g_{1}(u)\right)^{\prime} & =\delta u^{\delta-1} g_{2}(u-1), \quad u>\beta,
\end{array}
$$

and $A, B$, and $\beta$ are constants that depend on $\delta$. One might expect that an asymptotic relation similar to (3) holds as well in the case that $\frac{1}{2} \leq \delta<1$, and this is precisely what we shall prove. Our main result is the following. 
THEOREM. Let $Q$ be a set of primes satisfying (1) with $0<\delta<1$ and $A>1$. Then for any $\gamma$ with $0<\gamma<1-\delta$ there exists a constant $C$ such that

$$
\phi(x, y, Q)=x f(u) W(y)\left(1+O\left(\frac{1}{\log \log y}+\frac{\log (1+u)}{\log ^{\gamma} y}\right)\right)
$$

uniformly for $x \geq y \geq \exp \left(C(\log \log x)^{1 / \gamma}\right)$, where $u=\frac{\log x}{\log y}$ and $f$ is defined by (4).

We have made little effort to optimize the bound for the error term and the range of uniformity for $y$, and some improvements are possible (we will say more on this later). The primary importance of our theorem is for fixed $u$, since (2) already gives an asymptotic relation for $\phi(x, y, Q)$ in the case that $u \rightarrow \infty$. Note however that the bound on the relative error in our asymptotic expression is smaller than that of (2) in the case that $u$ tends slowly to infinity.

Our method of proof for the theorem is similar to that used by Hildebrand [5], and was also used by the authors in a previous paper [3] where we treated the case of sieving by the primes $>y$ that are in $Q$. The method is based on the "Buchstabtype" identity

$$
\begin{aligned}
\phi(x, y, Q) \log x= & \int_{1}^{x} \frac{\phi(t, y, Q)}{t} d t+\sum_{\substack{p^{m} \leq x \\
p \leq y \\
p \notin Q}} \phi\left(\frac{x}{p^{m}}, y, Q\right) \log p \\
& +\sum_{\substack{p^{m} \leq x \\
y<p \leq x}} \phi\left(\frac{x}{p^{m}}, y, Q\right) \log p .
\end{aligned}
$$

(Throughout this paper the letter $p$ denotes a prime number.)

Most sieve methods (see [4]) would make use of a Buchstab-type identity to estimate the sifting function $\phi\left(x, x^{1 / u}, Q\right)$ in terms of sifting functions $\phi\left(x, x^{1 / u_{1}}, Q\right)$ with $u_{1}>u$. The method of Hildebrand that we use in this paper works in the opposite direction, using estimates for smaller values of $u$ rather than larger values. In both cases we eventually arrive at a range of values of $u$ where it is possible to estimate the sifting function directly. In the case of $\phi(x, y, Q)$ when $Q$ consists of all primes, this is easy since we have $\phi(x, y, Q)=\pi(x)-\pi(y)$ if $1 \leq u \leq 2$. In the case that $Q$ has density less than one, the situation is somewhat more complicated, and in place of the prime number theorem we shall appeal to a result of Wirsing.

Specifically, it follows from [10, Satz 3] that if $Q$ satisfies (1) with $0<\delta<1$, then

$$
\phi(x, x, Q)=\frac{e^{(\delta-1) \gamma}}{\Gamma(1-\delta)} \frac{x}{\log x} \prod_{\substack{p \leq x \\ p \notin \notin}}\left(1-\frac{1}{p}\right)^{-1}\left(1+O\left(\frac{1}{\log \log 3 x}\right)\right) .
$$

In fact, [10] gives (6) from a much weaker hypothesis than (1). From (1) it may be proved in a standard manner (see [6, pp. 22 24]) that

$$
W(y)=\frac{B}{\log ^{\delta} y}\left(1+O\left(\frac{1}{\log y}\right)\right)
$$


for some constant $B$. Hence if $x<y$, then

$$
\begin{aligned}
\frac{W(y)}{W(x)} & =\prod_{\substack{x<p \leq y \\
p \in \bar{Q}}}\left(1-\frac{1}{p}\right) \\
& =\left(\frac{\log x}{\log y}\right)^{\delta}\left(1+O\left(\frac{1}{\log x}\right)\right) .
\end{aligned}
$$

Combining this with the result

$$
\prod_{p \leq x}\left(1-\frac{1}{p}\right)=\frac{e^{-\gamma}}{\log x}\left(1+O\left(\frac{1}{\log x}\right)\right),
$$

(see [6, p. 24]) it follows from (6) that

$$
\phi(x, y, Q)=x W(y) f(u)\left(1+O\left(\frac{1}{\log \log 3 x}\right)\right) .
$$

if $0<u \leq 1$.

It may be possible to improve on (6), and any improvement of the error term in (6) will result in an improvement of the error term in our main theorem when $u$ is small. In particular if $Q$ consists of the primes $\equiv 3(\bmod 4)$, then the error term $O\left(\frac{1}{\log \log x}\right)$ can probably be improved to $O\left(\frac{1}{\log x}\right)$, since one can apply essentially the same analytic argument as Landau (see [9, pp. 257-263]) for estimating the number of integers $\leq x$ that can be represented as the sum of two squares. Another improvement can be realized by applying the two step reduction process used in [3] to obtain a better bound for the error term when $u$ is large (or equivalently, when $y$ is small compared to $x$ ). This can be used to produce better short interval results for $\phi(x, y, Q)$.

2. Preliminary lemmas. Let $0<\delta<1$. We define the functions $f(u)=f_{\delta}(u)$ by the equation (4). These functions were studied previously by Iwaniec [7] (with slightly different notation). The properties that we require are summarized in the following lemma.

LEMMA 1. If $0<\delta<1$, then the function $f(u)$ satisfies

(i) $u f(u)=(1-\delta) \int_{u-1}^{u} f(t) d t+\int_{0}^{u-1} f(t) d t, u \geq 1$,

(ii) $f(u)=1+O\left(e^{-u}\right), u \geq 1$,

(iii) $f^{\prime}(u)=O\left(e^{-u}\right), u \geq 1$.

ProOF. If we differentiate both sides of (4), then we find that $u f^{\prime}(u)=$ $\delta f(u-1)-\delta f(u)$, for $u>1$. Now define

$$
g(u)=u f(u)-(1-\delta) \int_{u-1}^{u} f(t) d t-\int_{0}^{u-1} f(t) d t .
$$

Differentiating, we find that $g^{\prime}(u)=0$ for $u>1$. Hence $g$ is constant, and by letting $u$ tend to 1 , we see that the constant is zero. (ii) and (iii) were proved by Iwaniec [7]. 
LEMMA 2. If $y \leq x$, then

$$
\sum_{\substack{y<p^{m} \leq x \\ m \geq 2}} \phi\left(\frac{x}{p^{m}}, y, Q\right) \log p \ll x y^{-1 / 2} .
$$

ProOF. Let $\psi^{*}(t)=\sum_{\substack{p^{m} \leq t \\ m \geq 2}} \log p$. It follows from the prime number theorem that $\psi^{*}(t) \ll t^{1 / 2}$. Hence from the trivial estimate $\phi(t, y, Q) \leq t$ we obtain

$$
\begin{aligned}
\sum_{\substack{y<p^{m} \leq x \\
m \geq 2}} \phi\left(\frac{x}{p^{m}}, y, Q\right) \log p & \leq x \sum_{\substack{y<p^{m} \leq x \\
m \geq 2}} \frac{\log p}{p^{m}} \\
& =x \int_{y}^{x} \frac{d \psi^{*}(t)}{t} \\
& =\psi^{*}(x)-x \frac{\psi^{*}(y)}{y}+x \int_{y}^{x} \frac{\psi^{*}(t) d t}{t^{2}} \\
& \ll x^{1 / 2}+x \int_{y}^{x} t^{-3 / 2} d t
\end{aligned}
$$

which proves the result.

LEMMA 3. If $0<\varepsilon<0.5$ and $u \geq 1+\varepsilon$, then

$$
\sum_{y^{u-\varepsilon<p \leq y^{u}}} \phi\left(\frac{y^{u}}{p}, y, Q\right) \log p \ll \frac{y^{u}}{\log y^{u}}+\varepsilon y^{u} \log y .
$$

PROOF. Let $\theta(t)=\sum_{p \leq t} \log p$. It follows from the prime number theorem that $\theta(t)=t+\operatorname{tr}(t)$, where $r(t)=O\left(\log ^{-1} t\right)$. Hence

$$
\begin{aligned}
\sum_{y^{u-\varepsilon<p \leq y^{u}}} \phi\left(\frac{y^{u}}{p}, y, Q\right) \log p & \leq y^{u} \sum_{y^{u-\varepsilon<p \leq y^{u}}} \frac{\log p}{p} \\
& =y^{u} \int_{y^{u-\varepsilon}}^{y^{u}} \frac{d \theta(t)}{t} \\
& =y^{u} r\left(y^{u}\right)-y^{u} r\left(y^{u-\varepsilon}\right)+y^{u} \int_{y^{u-\varepsilon}}^{y^{u}} \frac{\theta(t) d t}{t^{2}} \\
& \ll \frac{y^{u}}{\log y^{u}}+\varepsilon y^{u} \log y .
\end{aligned}
$$

LEMMA 4. If $0<\alpha \leq 1, u \geq 1+\varepsilon$, and $0<\varepsilon<0.5$, then

$$
\begin{aligned}
\sum_{\substack{p^{m} \leq y^{\alpha} \\
p \notin Q}} \frac{\log p}{p^{m}} f\left(u-\frac{\log p^{m}}{\log y}\right)= & (1-\delta) \log y \int_{u-\alpha}^{u} f(t) d t \\
& +O\left(1+\varepsilon^{-\delta} \log ^{-A} y\right),
\end{aligned}
$$

where the implied $O$ constant may depend on $\alpha$. 
PROOF. We integrate by parts and replace $\psi\left(t, Q^{c}\right)$ by $(1-\delta) t+t R(t)$ to obtain

$$
\begin{aligned}
\sum_{\substack{p^{m} \leq y^{\alpha} \\
p \notin Q}} \frac{\log p}{p^{m}} f\left(u-\frac{\log p^{m}}{\log y}\right) & =\int_{2-}^{y^{\alpha}} \frac{f\left(u-\frac{\log t}{\log y}\right) d \psi\left(t, Q^{c}\right)}{t} \\
= & \frac{\psi\left(y^{\alpha}, Q^{c}\right) f(u-\alpha)}{y^{\alpha}} \\
& +\int_{u-\alpha}^{u-\frac{\log 2}{\log y}}\left\{f(w) \log y+f^{\prime}(w)\right\} y^{w-u} \psi\left(y^{u-w}, Q^{c}\right) d w \\
= & T_{1}+T_{2},
\end{aligned}
$$

where

$$
\begin{aligned}
T_{1} & =(1-\delta) f(u-\alpha)+(1-\delta) \int_{u-\alpha}^{u-\frac{\log 2}{\log y}}\left\{f(w) \log y+f^{\prime}(w)\right\} d w \\
& =(1-\delta) f\left(u-\frac{\log 2}{\log y}\right)+(1-\delta) \log y \int_{u-\alpha}^{u-\frac{\log 2}{\log y}} f(w) d w \\
& =(1-\delta) \log y \int_{u-\alpha}^{u} f(w) d w+O(1),
\end{aligned}
$$

and

$$
\begin{aligned}
T_{2}=f(u-\alpha) R\left(y^{\alpha}\right)+\int_{u-\alpha}^{u-\frac{\log 2}{\log y}}\left\{f(w) \log y+f^{\prime}(w)\right\} R\left(y^{u-w}\right) d w \\
\ll_{\alpha} \varepsilon^{-\delta} \log ^{-A} y+\log ^{1-A} y \int_{u-\alpha}^{u-\frac{\log 2}{\log y}} \frac{f(w) d w}{(u-w)^{A}} \\
\quad+\log ^{-A} y \int_{\varepsilon}^{u-\frac{\log 2}{\log y}} \frac{\left|f^{\prime}(w)\right| d w}{(u-w)^{A}} .
\end{aligned}
$$

Let $u_{1}=u-\frac{\log 2}{2 \log y}-\frac{\alpha}{2}$, the midpoint of the interval of integration. Then

$$
\begin{aligned}
\int_{u-\alpha}^{u-\frac{\log 2}{\log y}} \frac{f(w) d w}{(u-w)^{A}} & \ll\left(u-u_{1}\right)^{-A} \int_{u-\alpha}^{u_{1}} f(w) d w+\int_{u_{1}}^{u-\frac{\log 2}{\log y}} \frac{d w}{(u-w)^{A}} \\
& \ll 1+\log ^{A-1} y .
\end{aligned}
$$

We will now consider several cases for estimating the second integral of (8). If $u \leq 1+\frac{\log 2}{\log y}$, then we have

$$
\begin{aligned}
\int_{\varepsilon}^{u-\frac{\log 2}{\log y} \frac{\left|f^{\prime}(w)\right| d w}{(u-w)^{A}}} & \ll \int_{\varepsilon}^{1 / 2} w^{-\delta-1} d w+\int_{1 / 2}^{u-\frac{\log 2}{\log y}}(u-w)^{-A} d w \\
& \ll \varepsilon^{-\delta}+\log ^{A-1} y .
\end{aligned}
$$

If instead we have $1<u-\frac{\log 2}{\log y} \leq 2$, then we have

$$
\int_{\varepsilon}^{u-\frac{\log 2}{\log y}} \frac{\left|f^{\prime}(w)\right| d w}{(u-w)^{A}}=\int_{\varepsilon}^{1}+\int_{1}^{u-\frac{\log 2}{\log y}} .
$$


The first of these integrals can be treated as in the previous case, and the second satisfies

$$
\int_{1}^{u-\frac{\log 2}{\log y}} \frac{\left|f^{\prime}(w)\right|}{(u-w)^{A}} d w \ll \log ^{A} y \int_{1}^{2}\left|f^{\prime}(w)\right| d w \ll \log ^{A} y .
$$

If finally we have $u-\frac{\log 2}{\log y}>2$, then we can estimate the contribution from $\varepsilon<$ $w<1.5$ as before, and by Lemma 1 the remainder is dominated by

$$
\int_{1.5}^{u-\frac{\log 2}{\log y}} \frac{e^{-w}}{(u-w)^{A}} d w \ll \log ^{A} y
$$

This completes the proof.

LEMMA 5. If $0<\varepsilon<0.5$ and $u \geq 1+\varepsilon$, then

$$
\begin{aligned}
\sum_{y<p \leq y^{u-\varepsilon}} \frac{\log p}{p} f & \left(u-\frac{\log p}{\log y}\right) \\
& =\log y \int_{0}^{u-1} f(t) d t+O\left(\varepsilon^{1-\delta} \log y+\varepsilon^{-\delta} \log ^{-A} y+\log ^{1-A} y\right) .
\end{aligned}
$$

PrOOF. With $\theta(t)$ and $r(t)$ as in the proof of Lemma 3, integration by parts yields

$$
\sum_{y<p \leq y^{u-\varepsilon}} \frac{\log p}{p} f\left(u-\frac{\log p}{\log y}\right)=\int_{y}^{y^{u-\varepsilon}} \frac{f\left(u-\frac{\log t}{\log y}\right)}{t} d \theta(t)=T_{1}+T_{2},
$$

where

$$
\begin{aligned}
T_{1} & =f(\varepsilon)-f(u-1)+\int_{y}^{y^{u-\varepsilon}}\left\{f\left(u-\frac{\log t}{\log y}\right)+\frac{f^{\prime}\left(u-\frac{\log t}{\log y}\right)}{\log y}\right\} \frac{d t}{t} \\
& =\log y \int_{\varepsilon}^{u-1} f(w) d w,
\end{aligned}
$$

and

$$
\begin{aligned}
T_{2}=r\left(y^{u-\varepsilon}\right) f(\varepsilon)-r(y) f(u-1) \\
\quad+\int_{y}^{y^{u-\varepsilon}}\left\{f\left(u-\frac{\log t}{\log y}\right)+\frac{f^{\prime}\left(u-\frac{\log t}{\log y}\right)}{\log y}\right\} \frac{r(t) d t}{t} .
\end{aligned}
$$

Note that

$$
\begin{aligned}
T_{1}-\log y \int_{0}^{u-1} f(w) d w & =-\log y \int_{0}^{\varepsilon} f(w) d w \\
& =O\left(\varepsilon^{1-\delta} \log y\right) .
\end{aligned}
$$

The prime number theorem implies that $r(t) \ll \log ^{-A} t$, so that

$$
\begin{aligned}
T_{2} \ll & \varepsilon^{-\delta} \log ^{-A} y+\log ^{1-A} y \int_{0}^{u-1} \frac{f(w)}{(u-w)^{A}} d w \\
& +\log ^{-A} y \int_{\varepsilon}^{u-1} \frac{\left|f^{\prime}(w)\right|}{(u-w)^{A}} d w .
\end{aligned}
$$


If $u \geq 2$, then the first integral satisfies

$$
\begin{aligned}
\int_{0}^{u-1} \frac{f(w)}{(u-w)^{A}} d w & \ll \int_{0}^{1} f(w) d w+\int_{1}^{u-1} \frac{d w}{(u-w)^{A}} \\
& \ll 1 .
\end{aligned}
$$

If $u<2$, then the first integral of (9) is bounded by $\int_{0}^{1} f(w) d w \ll 1$.

For $u \geq 3$, we use Lemma 1 to estimate the second integral of (9) as

$$
\begin{aligned}
\int_{\varepsilon}^{u-1} \frac{\left|f^{\prime}(w)\right|}{(u-w)^{A}} d w & \ll \int_{\varepsilon}^{2}\left|f^{\prime}(w)\right| d w+\int_{2}^{u-1} e^{-w} d w \\
& \ll \varepsilon^{-\delta}+1 .
\end{aligned}
$$

If $1+\varepsilon \leq u \leq 3$, then this last estimate may be proved in essentially the same manner, and combining these estimates proves the lemma.

3. Proof of the theorem. In order to now prove the theorem, we first give a proof of (5). Let $P(y)$ denote the product of the primes belonging to $Q$ that do not exceed $y$. Then

$$
\begin{aligned}
\sum_{\substack{n \leq x \\
(n, P(y))=1}} \log n= & \sum_{\substack{n \leq x \\
(n, P(y))=1}} \sum_{p^{m} \mid n} \log p \\
= & \sum_{\substack{p^{m} \leq x \\
p \nmid P(y)}} \log p \sum_{\substack{n \leq x \\
n \equiv 0\left(\bmod p^{m}\right) \\
(n, P(y))=1}} 1 \\
= & \sum_{\substack{p^{m} \leq x \\
p \leq y \\
p \notin Q}} \phi\left(\frac{x}{p^{m}}, y, Q\right) \log p \\
& +\sum_{\substack{p^{m} \leq x \\
y<p \leq x}} \phi\left(\frac{x}{p^{m}}, y, Q\right) \log p .
\end{aligned}
$$

On the other hand we can integrate by parts to get

$$
\begin{aligned}
\sum_{\substack{n \leq x \\
(n, P(y))=1}} \log n & =\int_{1}^{x} \log t d \phi(t, y, Q) \\
& =\phi(x, y, Q) \log x-\int_{1}^{x} \frac{\phi(t, y, Q)}{t} d t
\end{aligned}
$$

and this proves (5).

For $u>0$ and $y \geq 1.5$, we define $\Delta(u, y)$ by the relation

$$
\phi\left(y^{u}, y, Q\right)=y^{u} W(y) f(u)(1+\Delta(u, y)) .
$$

Furthermore for $0<\varepsilon<0.5$ and $u \geq \varepsilon$, we define

$$
\begin{aligned}
\Delta^{*}(u, y) & =\Delta^{*}(u, y, \varepsilon)=\sup _{\varepsilon \leq u^{\prime} \leq u}\left|\Delta\left(u^{\prime}, y\right)\right| \\
\Delta^{* *}(u, y) & =\sup _{0 \leq u^{\prime} \leq u}\left|\Delta\left(u^{\prime}, y\right)\right| .
\end{aligned}
$$


The initial conditions (7) imply that

$$
\Delta(u, y) \ll \frac{1}{\log \log \left(3 y^{u}\right)}
$$

uniformly for $0<u \leq 1$. Let $\gamma$ be fixed, with $0<\gamma<1-\delta$, and define $\varepsilon$ by

$$
\varepsilon=\log ^{-\delta-\gamma} y \text {. }
$$

In order to prove the theorem it suffices to prove that

$$
\Delta^{*}(u, y) \ll \frac{1}{\log \log 3 y}+\frac{\log (1+u)}{\log ^{\gamma} y},
$$

uniformly for $y \geq 1.5$ and $u \geq \frac{1}{2}$. Without loss of generality we may assume that $y$ is sufficiently large. It follows from (10) that if $0.5 \leq u \leq 1$, then (11) is satisfied. For $u>1$, we will now use the identity (5) to estimate $\Delta^{*}(u, y)$ in terms of $\Delta^{*}\left(u^{\prime}, y\right)$ with some $\frac{1}{2} \leq u^{\prime} \leq 1$.

Let

$$
m=\inf _{t \geq 1 / 2} f(t), \quad M=\sup _{t \geq 1 / 2} f(t) .
$$

It follows from Lemma 1 that $0<m<M<\infty$. For $u \geq 1$, we now define

$$
\begin{aligned}
\lambda & =\min \left\{\frac{1}{2}, \frac{m}{2(1-\delta) M}\right\}, \\
\alpha_{1} & =\frac{1-\delta}{u f(u)} \int_{u-\lambda}^{u} f(t) d t, \\
\alpha_{2} & =\frac{1-\delta}{u f(u)} \int_{u-1}^{u-\lambda} f(t) d t, \\
\alpha_{3} & =\frac{1}{u f(u)} \int_{0}^{u-1} f(t) d t .
\end{aligned}
$$

It follows from Lemma 1 that $\alpha_{1}+\alpha_{2}+\alpha_{3}=1$, and clearly by the choice of $\lambda$ we have $\alpha_{1} \leq \frac{1}{2}$.

Let $u \geq 1+\varepsilon$. We begin by rewriting (5) in terms of $\Delta$ to obtain

(12) $1+\Delta(u, y)=R_{1}+R_{2}$

$$
\begin{aligned}
& +\frac{1}{f(u) \log y^{u}} \sum_{\substack{p^{m} \leq y \\
p \notin Q Q}} \frac{\log p}{p^{m}} f\left(u-\frac{\log p^{m}}{\log y}\right)\left(1+\Delta\left(u-\frac{\log p^{m}}{\log y}, y\right)\right) \\
& +\frac{1}{f(u) \log y^{u}} \sum_{y<p \leq y^{u-\varepsilon}} \frac{\log p}{p} f\left(u-\frac{\log p}{\log y}\right)\left(1+\Delta\left(u-\frac{\log p}{\log y}, y\right)\right) \\
& +\frac{1}{f(u) y^{u} \log y^{u}} \int_{1}^{y^{u}} f\left(\frac{\log t}{\log y}\right)\left(1+\Delta\left(\frac{\log t}{\log y}, y\right)\right) d t,
\end{aligned}
$$

where

$$
\begin{aligned}
& R_{1}=\frac{1}{W(y) f(u) y^{u} \log y^{u}} \sum_{\substack{y<p^{m} \leq y^{u} \\
p \leq y \Rightarrow p \notin \\
m \geq 2}} \phi\left(\frac{y^{u}}{p^{m}}, y, Q\right) \log p, \\
& R_{2}=\frac{1}{W(y) f(u) y^{u} \log y^{u}} \sum_{y^{u-\varepsilon<p \leq y^{u}}} \phi\left(\frac{y^{u}}{p}, y, Q\right) \log p .
\end{aligned}
$$


From this we obtain for $u \geq 1+\varepsilon$ that

$$
\begin{aligned}
|\Delta(u, y)| \leq & \left|R_{1}\right|+\left|R_{2}\right|+\left(1+\Delta^{* *}(u, y)\right)\left|R_{3}\right| \\
& +\left|R_{4}\right|+\left|R_{5}\right|+\left|R_{6}\right| \\
& +\frac{\Delta^{*}(u, y)}{f(u) \log y^{u}} \sum_{\substack{p^{m} \leq y^{\lambda} \\
p \notin Q}} \frac{\log p}{p^{m}} f\left(u-\frac{\log p^{m}}{\log y}\right) \\
& +\frac{\Delta^{*}(u-\lambda, y)}{f(u) \log y^{u}} \sum_{y^{\lambda}<p^{m} \leq y} \frac{\log p}{p^{m}} f\left(u-\frac{\log p^{m}}{\log y}\right) \\
& +\frac{\Delta^{*}(u-1, y)}{f(u) \log y^{u}} \sum_{y<p \leq y^{u-\varepsilon}} \frac{\log p}{p} f\left(u-\frac{\log p}{\log y}\right) \\
\leq & \alpha_{1} \Delta^{*}(u, y)+\alpha_{2} \Delta^{*}(u-\lambda, y)+\alpha_{3} \Delta^{*}(u-1, y) \\
& +O\left(\left(1+\Delta^{* *}(u, y)\right) \sum_{i=1}^{6}\left|R_{i}\right|\right)
\end{aligned}
$$

where

$$
\begin{aligned}
& R_{3}=\frac{1}{f(u) y^{u} \log y^{u}} \int_{1}^{y^{u}} f\left(\frac{\log t}{\log y}\right) d t \\
& R_{4}=\frac{1}{f(u) \log y^{u}} \sum_{\substack{p^{m} \leq y^{\lambda} \\
p \notin Q}} \frac{\log p}{p^{m}} f\left(u-\frac{\log p^{m}}{\log y}\right)-\alpha_{1}, \\
& R_{5}=\frac{1}{f(u) \log y^{u}} \sum_{y^{\lambda}<p^{m} \leq y} \frac{\log p}{p^{m}} f\left(u-\frac{\log p^{m}}{\log y}\right)-\alpha_{2}, \\
& R_{6}=\frac{1}{f(u) \log y^{u}} \sum_{y<p \leq y^{u-\varepsilon}} \frac{\log p}{p} f\left(u-\frac{\log p}{\log y}\right)-\alpha_{3} .
\end{aligned}
$$

The term $R_{1}$ is $\ll(u \log y)^{-1}$ by Lemma 2 , and Lemma 3 yields

$$
\begin{aligned}
\left|R_{2}\right| & \ll(u \log y)^{-1}+\varepsilon u^{-1} \log ^{\delta} y \\
& \ll u^{-1} \log ^{-\gamma} y .
\end{aligned}
$$

The term $R_{3}$ satisfies

$$
\begin{aligned}
\left|R_{3}\right| & =\frac{1}{f(u) y^{u} u} \int_{0}^{u} y^{w} f(w) d w \\
& \ll \frac{1}{y^{u} u}\left(y^{1 / 2} \int_{0}^{1 / 2} w^{-\delta} d w+\int_{1 / 2}^{u} y^{w} d w\right) \\
& \ll(u \log y)^{-1} .
\end{aligned}
$$

Lemma 4 yields the estimate

$$
\left|R_{4}\right|+\left|R_{5}\right| \ll \frac{1+\varepsilon^{-\delta} \log ^{-A} y}{u \log y} \ll(u \log y)^{-1},
$$


and Lemma 5 yields the estimate

$$
\begin{aligned}
\left|R_{6}\right| & \ll\left(\frac{\varepsilon^{1-\delta} \log y+\varepsilon^{-\delta} \log ^{-A} y+\log ^{1-A} y}{u \log y}\right) \\
& \ll u^{-1} \log ^{-\gamma} y .
\end{aligned}
$$

From (10) we may deduce that $\Delta^{* *}(u, y) \leq C+\Delta^{*}(u, y)$ for $u \geq \varepsilon$ and some $C>0$. Combining these estimates with $(13)$ and the fact that $\Delta^{*}$ is nondecreasing gives

$$
\begin{aligned}
|\Delta(u, y)| \leq & \alpha_{1} \Delta^{*}(u, y)+\left(\alpha_{2}+\alpha_{3}\right) \Delta^{*}(u-\lambda, y) \\
& +O\left(\frac{1+\Delta^{*}(u, y)}{u \log ^{\gamma} y}\right) .
\end{aligned}
$$

Since $\alpha_{1} \leq 0.5$ we have

$$
\begin{aligned}
0.5\left(\Delta^{*}(u, y)+\Delta^{*}(u-\lambda, y)\right) & -\alpha_{1} \Delta^{*}(u, y)-\left(\alpha_{2}+\alpha_{3}\right) \Delta^{*}(u-\lambda, y) \\
& =\left(0.5-\alpha_{1}\right)\left(\Delta^{*}(u, y)-\Delta^{*}(u-\lambda, y)\right),
\end{aligned}
$$

and the latter quantity is clearly nonnegative. Hence (14) gives

$$
|\Delta(u, y)| \leq 0.5 \Delta^{*}(u, y)+0.5 \Delta^{*}(u-\lambda, y)+O\left(\frac{1+\Delta^{*}(u, y)}{u \log ^{\gamma} y}\right) \text {. }
$$

The same argument as in [5, pp. 300-301] gives

$$
\Delta^{*}(u, y) \leq \Delta^{*}(u-\lambda, y)+O\left(\frac{1+\Delta^{*}(u, y)}{u \log ^{\gamma} y}\right)
$$

uniformly for $u \geq 1+\varepsilon$. We iterate this bound to obtain

$$
\Delta^{*}(u, y) \leq \Delta^{*}\left(u_{0}, y\right)+O\left(\frac{\left(1+\Delta^{*}(u, y)\right) \log (1+u)}{\log ^{\gamma} y}\right)
$$

for some $u_{0}$ with $1+\varepsilon-\lambda \leq u_{0}<1+\varepsilon$. From (15) we now obtain

$$
\Delta^{*}\left(u_{0}, y\right) \leq \Delta^{*}(1+\varepsilon, y) \leq \Delta^{*}(1+\varepsilon-\lambda, y)+O\left(\frac{1+\Delta^{*}(1+\varepsilon, y)}{\log ^{\gamma} y}\right) .
$$

Since $1+\varepsilon-\lambda<1$ for $y$ sufficiently large, this gives

$$
\Delta^{*}\left(u_{0}, y\right) \ll \frac{1}{\log \log y}+\frac{1+\Delta^{*}(u, y)}{\log ^{\gamma} y} .
$$

The result now follows from (16), provided $\frac{\log (1+u)}{\log \gamma y}$ is sufficiently small. The latter condition is satisfied if the constant $C$ of the theorem is chosen sufficiently large.

\section{REFERENCES}

1. N. G. de Bruijn, On the number of uncancelled elements in the sieve of Eratosthenes, Indag. Math. 12 (1950), 247-256.

2. A. A. Buchstab, Asymptotic estimates of a general number-theoretic function (Russian, German summary), Mat. Sbornik 44 (1937), 1239-1246.

3. D. A. Goldston and Kevin S. McCurley, Sieving the positive integers by large primes, J. Number Theory 28 (1988), 94-115.

4. H. Halberstam and H.-E. Richert, Sieve methods, Academic Press, London, 1974. 
5. A. Hildebrand, On the number of positive integers $\leq x$ and free of prime factors $>y$, J. Number Theory 22 (1986), 289-307.

6. A. E. Ingham, The distribution of prime numbers, Hafner, New York, 1971.

7. H. Iwaniec, The sieve of Eratosthenes-Legendre, Ann. Scuola Norm. Sup. Pisa Cl. Sci. 4 (1977), 257-268.

8. H. Iwaniec, Rosser's Sieve, Acta Arith. 36 (1980), 171--202.

9. W. J. LeVeque, Topics in number theory, Addison-Wesley, Reading, Mass., 1956.

10. E. Wirsing, Über die Zahlen, deren Primteiler einer gegeben Menge angehören, Arch. Math. (Basel) 7 (1956), 263-272.

Department of Mathematics and Computer Science, San Jose State UniverSITY, SAN JOSE, CALIFORNIA 95192

Department of Mathematics, University of Southern California, Los ANGELES, CALIFORNIA 90089-1113 